\title{
Subcutaneous metastasis arising from gastric cancer: A case report
}

\author{
TSUTOMU NAMIKAWA ${ }^{1}$, ERI MUNEKAGE ${ }^{1}$, MASAYA MUNEKAGE $^{1}$, HIROMICHI MAEDA $^{2}$, \\ TOMOAKI YATABE $^{3}$, HIROYUKI KITAGAWA ${ }^{1}$, MICHIYA KOBAYASHI ${ }^{2,4}$ and KAZUHIRO HANAZAKI ${ }^{1}$ \\ ${ }^{1}$ Department of Surgery; ${ }^{2}$ Cancer Treatment Center; Departments of ${ }^{3}$ Anesthesiology and ${ }^{4}$ Human Health \\ and Medical Sciences, Kochi Medical School, Nankoku, Kochi 783-8505, Japan
}

Received November 7, 2016; Accepted January 10, 2017

DOI: $10.3892 / \mathrm{mco} .2017 .1175$

\begin{abstract}
A 59-year-old man was referred to the Kochi Medical School Hospital due to left shoulder pain. Physical examination revealed a nodular, painful mass lesion in the subcutaneous tissue of the right chest wall, with a diameter of $\sim 2 \mathrm{~cm}$. Esophagogastroduodenoscopy revealed a large ulcerated tumor in the lower gastric body near the lesser curvature, and biopsy specimens of the gastric and humeral lesions revealed poorly differentiated adenocarcinoma. Abdominal computed tomography revealed multiple low-density lesions in the liver and a well-defined, $2.2-\mathrm{cm}$ mass in the subcutaneous tissue of the right chest wall. The diagnosis was advanced gastric cancer with metastases to the liver, bone and skin, and systemic chemotherapy with radiotherapy for the bone metastasis was recommended. The patient also underwent distal gastrectomy to control bleeding from the primary gastric cancer lesion due to unresolved anemia, not improving by blood transfusion, and a low hemoglobin level. The subcutaneous tumor in the right chest wall was resected at the same time, to alleviate the pain caused by the mass. Macroscopic examination of the resected specimen revealed a well-circumscribed, solid tumor, measuring $2.2 \times 2.1 \mathrm{~cm}$, with a firm consistency. Microscopic examination showed infiltration of poorly differentiated adenocarcinoma cells into the subcutaneous tissue. Subcutaneous metastasis from gastric cancer is a rare manifestation, with a reported incidence of $0.8-1.0 \%$. Cutaneous metastasis of cancer generally appears late in the course of the disease. Surgical resection of metastatic skin tumors is occasionally undertaken as palliative treatment to improve the patient's quality of life by controlling severe symptoms, including pain and hemorrhage.
\end{abstract}

Correspondence to: Dr Tsutomu Namikawa, Department of Surgery, Kochi Medical School, Kohasu, Oko-cho, Nankoku, Kochi 783-8505, Japan

E-mail: tsutomun@kochi-u.ac.jp

Key words: gastric cancer, adenocarcinoma, subcutaneous metastases

\section{Introduction}

Gastric cancer is the third most commonly diagnosed cancer worldwide, and a leading cause of cancer-related mortality. The most common metastatic sites of gastric cancer are the lymph nodes, liver and peritoneum, while it may be associated with multiple sites of metastasis. Subcutaneous metastasis from gastric cancer is a rare manifestation, with a reported incidence of $0.8-1.0 \%$ (1-3). We herein report a case of subcutaneous metastasis from gastric cancer in a patient who underwent surgical resection and systemic chemotherapy.

\section{Case report}

A 59-year-old man was referred to the Kochi Medical School Hospital (Nankoku, Japan) in February, 2016, due to left shoulder pain. Physical examination revealed a nodular painful mass lesion in the subcutaneous tissue of the right chest wall, measuring $\sim 2 \mathrm{~cm}$ in diameter (Fig. 1). X-ray and magnetic resonance imaging revealed an osteolytic tumor in the proximal region of the left humerus. Esophagogastroduodenoscopy revealed a large ulcerated tumor in the lower gastric body near the lesser curvature, and biopsy specimens from the gastric and humeral lesions revealed poorly differentiated adenocarcinoma (Fig. 2). Abdominal computed tomography examination revealed multiple low-density lesions in the liver and a well-defined, 2.2-cm mass in the subcutaneous tissue of the right chest wall (Fig. 3).

The diagnosis was advanced gastric cancer with metastases to the liver, bone and skin, and systemic chemotherapy with radiotherapy were recommended for the bone metastasis. The patient also underwent distal gastrectomy to control bleeding from the primary gastric cancer lesion due to unresolved anemia, not improving by blood transfusion, and a low hemoglobin level (7.3 g/dl; normal range, 13.7-16.8 g/dl). The subcutaneous tumor in the right chest wall was resected at the same time, in order to alleviate the pain caused by the mass. Macroscopic examination of the resected specimen revealed a well-circumscribed, solid tumor, measuring $2.2 \times 2.1 \mathrm{~cm}$, with a firm consistency (Fig. 4). Microscopic examination revealed infiltration of the subcutaneous tissue by poorly differentiated adenocarcinoma cells. The patient received chemotherapy with oxaliplatin and oral fluoropyrimidine (S-1). However, the liver metastases and peritoneal carcinomatosis progressed, and the patient succumbed to the disease 6 months after the diagnosis. 


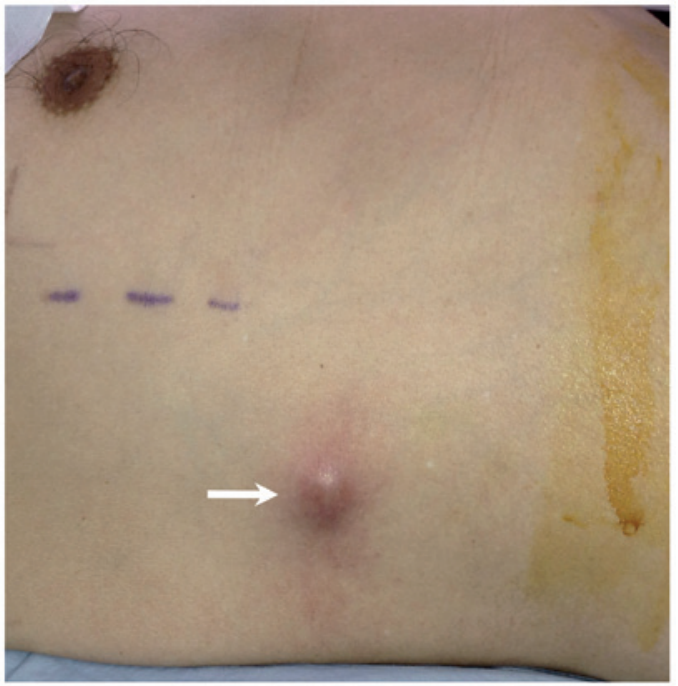

Figure 1. Cutaneous metastatic lesion in the right chest wall presenting as an elevated mass with erythema of the overlying skin (arrow).

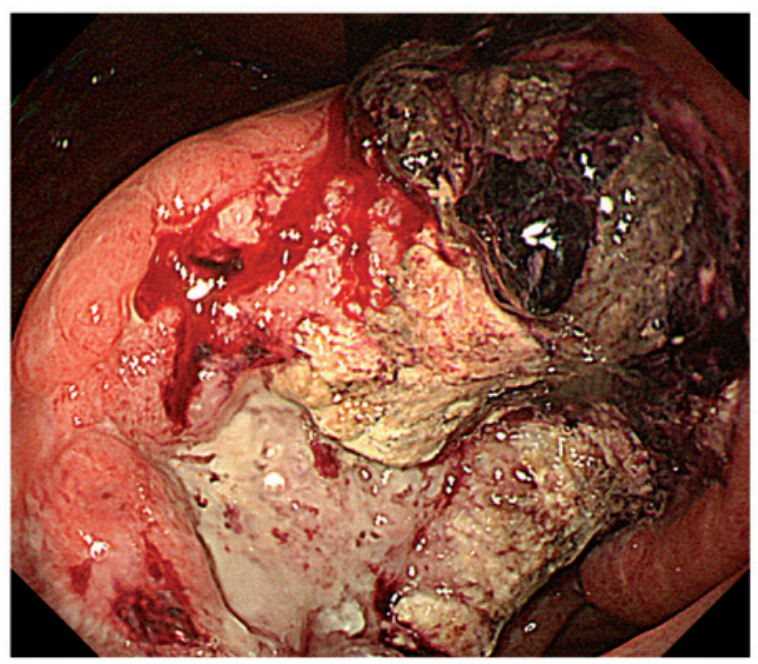

Figure 2. Esophagogastroduodenoscopy showing a giant ulcerated, oozing tumor located in the lower part of the stomach body, near the lesser curvature.

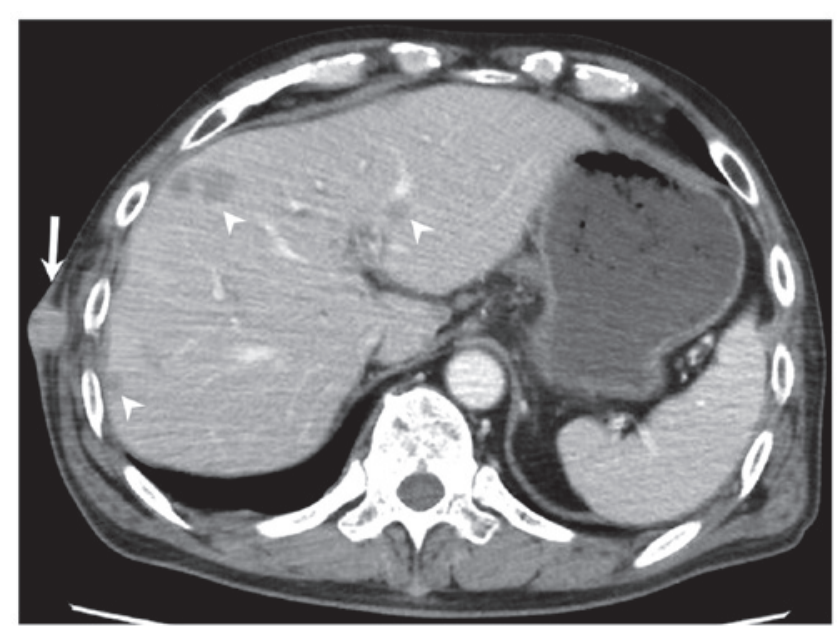

Figure 3. Abdominal computed tomography examination revealed a well-circumscribed, smooth-bordered, high-density mass in the subcutaneous tissue of the right chest wall (arrow), and low-density liver metastases (arrowheads).

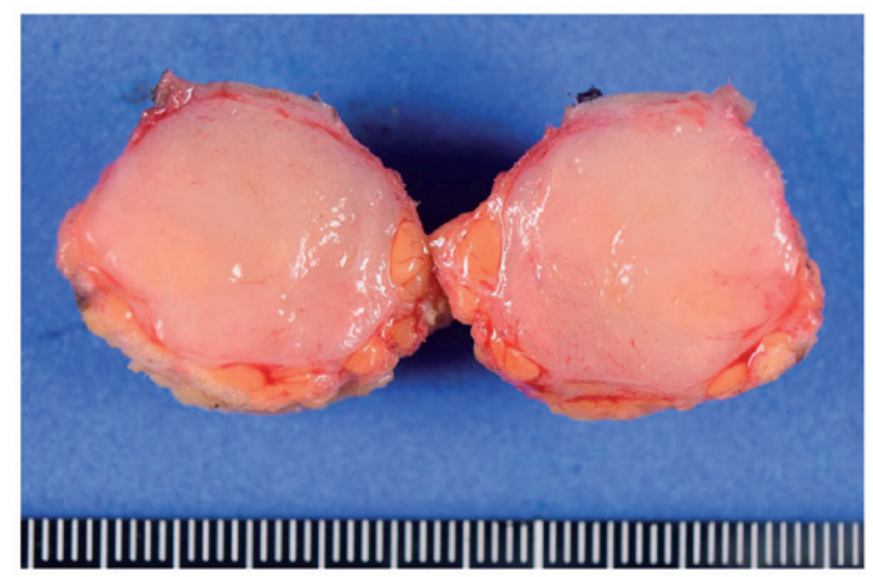

Figure 4. Macroscopic appearance of the surgically resected chest wall lesion, showing a well-circumscribed, solid tumor, measuring $2.2 \times 2.1 \mathrm{~cm}$.

\section{Discussion}

The most commonly reported origins of cutaneous metastasis are lung, breast and colon cancer, melanoma, squamous cell carcinoma of the oral cavity and renal cell carcinoma $(2,4)$. Lymphatic and hematogenous spread have been suggested as possible mechanisms for metastasis to the skin as well as to other organs $(4,5)$, although most metastases of primary cancer are generally considered to progress, in a stepwise manner, from locoregional to distant lesions (6).

Cutaneous metastasis of cancer generally appears late in the course of the disease. Therefore, the mean survival time of patients with skin metastases is only a few months and, in cases of metastatic gastric tumors, treatment usually consists of systemic therapy rather than surgery (6). However, as in the present case, surgical resection of metastatic skin tumors is occasionally undertaken as palliative treatment to improve the patient's quality of life by controlling severe symptoms such as pain and hemorrhage.

\section{References}

1. Kawai S, Nishida T, Hayashi Y, Ezaki H, Yamada T, Shinzaki S, Miyazaki M, Nakai K, Yakushijin T, Watabe K, et al: Choroidal and cutaneous metastasis from gastric adenocarcinoma. World $\mathrm{J}$ Gastroenterol 19: 1485-1488, 2013.

2. Hu SC, Chen GS, Wu CS, Chai CY, Chen WT and Lan CC: Rates of cutaneous metastases from different internal malignancies: Experience from a Taiwanese medical center. J Am Acad Dermatol 60: 379-387, 2009.

3. Kairouani M, Perrin J, Dietemann-Barabinot A, Diab R and Ruck S: Cutaneous metastasis revealing a relapse of gastric linitis: Another case. Int J Surg Case Rep 4: 185-187, 2013.

4. Alcaraz I, Cerroni L, Rütten A, Kutzner H and Requena L: Cutaneous metastases from internal malignancies: A clinicopathologic and immunohistochemical review. Am J Dermatopathol 34: 347-393, 2012.

5. Avgerinou G, Flessas I, Hatziolou E, Zografos G, Nitsios I, Zagouri F, Katsambas A and Kanitakis J: Cutaneous metastasis of signet-ring gastric adenocarcinoma to the breast with unusual clinicopathological features. Anticancer Res 31: 2373-2378, 2011.

6. Namikawa T and Hanazaki K: Clinicopathological features and treatment outcomes of metastatic tumors in the stomach. Surg Today 44: 1392-1399, 2014. 